\title{
Rectificación a la primera cita de Flebotomos (Diptera, Psychodidae) en la Península Ibérica
}

\author{
C. M. León Sanz (*), F. Collantes (*) y E. Martínez-Ortega (*)
}

En 1909 Czerny \& Strobl, a partir de una serie de ejemplares de flebotomos capturados por Lauffer en Fte. El Tejar, El Escorial (Madrid), identificaron la especie P. papatasi (Scopoli, 1786), siendo ésta la primera cita de flebotomos para la Península Ibérica. Esta cita indica textualmente: "Phlebotomus papatasi Scop. Escorial, Haüfig (L.)". La inicial, entre paréntesis, hace mención a Lauffer y los autores califican la especie de "frecuente". Esta cita se ha mantenido como la primera mención de flebotomos en España a lo largo de todas las revisiones efectuadas hasta la fecha.

Habiendo tenido ocasión de revisar los ejemplares de Lauffer, depositados en el Museo Nacional de Ciencias Naturales (CSIC), se vio que de la serie de seis ejemplares allí depositados, tan solo uno se encontraba identificado, mientras que en toda la serie se indicaba la misma localidad de captura y a Lauffer como recolector de dichos ejemplares. Dado que Czerny \& Strobl (1909) citan la especie como frecuente, debemos suponer que asumían que todos los ejemplares pertenecían a la especie que ellos habían identificado, más aún considerando que en aquella fecha tan solo dos especies de flebotomos habían sido descritas: Phlebotomus papatasi y Sergentomyia minuta (Rondani, 1843).

Los ejemplares de esta captura se hallaban montados en seco, con lo cual el estudio microscópico de la genitalia de los mismos era imposible, aunque imprescindible para poder confirmar la correcta identificación de los mismos. Por ello, se hidrataron durante doce horas en agua destilada y fueron montados posteriormente para su identificación microscópica entre porta y cubreobjetos, usando como medio de montaje el líquido de Hoyer.

Las especies obtenidas han sido las siguientes:

- Phlebotomus ariasi Tonnoir, 1921, 4 hembras. Entre ellas el ejemplar identificado como $P$. papatasi por Czerny \& Strobl (1909).
- Phlebotomus perniciosus Newstead, 1911, 2 hembras.

Por tanto, la primera cita de flebotomos en la Península Ibérica corresponde a una hembra de la especie $P$. ariasi, identificada erróneamente como P. papatasi en 1908.

Así mismo, la primera captura de P. perniciosus en la Península corresponde al material obtenido por Lauffer, en el que capturó dos hembras de esta especie.

En relación con lo anterior, la primera identificación correcta que cita la presencia de $P$. papatasi en la Península Ibérica corresponde a França (1913), a partir de ejemplares capturados en Portugal, y la primera cita de esta especie en España se debe a Pittaluga \& De Buen (1917).

Los autores agradecen al Museo Nacional de Ciencias Naturales de Madrid el préstamo del material utilizado en este estudio.

\section{Referencias}

Czerny, L. \& Strobl, G., 1909. Spanische Dipteren III. Beitrag. Z. B. Ges., 59: 121-301.

França, C., 1913. Phlebotomus papatasi (Scopoli) et fièbre à Pappataci au Portugal. Bull. Soc. Pathol. Exot., 6: 123-124.

Pittaluga, G. \& De Buen, S., 1917. Notas sobre los dípteros del género Phlebotomus en España. Bol. Inst. Nac. Hig. Alfonso XIII, 13: 1-9.

* Dpto. Biología Animal (Zoología), Facultad de Biología, Universidad de Murcia. 30071 Murcia (España). 